\title{
Topological Insights into Mutant Huntingtin Exon 1 and PolyQ Aggregates by Cryo-Electron Tomography
}

Jesus Galaz-Montoya ( $\nabla$ jgalaz@gmail.com )

Stanford University https://orcid.org/0000-0002-0113-9325

\section{Sarah Shahmoradian}

Laboratory of Biomolecular Research, Paul Scherrer Institute

Koning Shen

Stanford University

Judith Frydman

Stanford University https://orcid.org/0000-0003-2302-6943

Wah Chiu

Stanford University https://orcid.org/0000-0002-8910-3078

\section{Article}

Keywords: HD, mHTT, CS, EMAN2

Posted Date: December 21st, 2020

DOI: https://doi.org/10.21203/rs.3.rs-125810/v1

License: (c) (1) This work is licensed under a Creative Commons Attribution 4.0 International License.

Read Full License

Version of Record: A version of this preprint was published at Communications Biology on July 8th, 2021. See the published version at https://doi.org/10.1038/s42003-021-02360-2. 
Topological Insights into Mutant Huntingtin Exon 1 and PolyQ Aggregates by

Jesús G. Galaz-Montoya ${ }^{1 \ddagger}$, Sarah H. Shahmoradian ${ }^{2^{*}}$, Koning Shen ${ }^{3^{*} \dagger}$, Judith

Frydman ${ }^{3}$, Wah Chiu $^{1,4 \ddagger}$

$5{ }^{1}$ Department of Bioengineering and James H. Clark Center, Stanford University,

6 Stanford, CA 94305, United States of America

$7 \quad 2$ Department of Biology and Chemistry, Laboratory of Biomolecular Research, Paul

8 Scherrer Institute, Villigen, Switzerland

$9{ }^{3}$ Department of Biology, Stanford University, Stanford, CA 94305, United States of

10 America

114 Division of CryoEM and Bioimaging, SSRL, SLAC National Accelerator Laboratory,

12 Menlo Park, CA 94025, United States of America

13

$14 *$ Equal contribution

15 † Current address: Department of Molecular and Cell biology, University of California,

16 Berkeley, CA 94720, United States

17 ‡ Correspondence to: Jesús G. Galaz-Montoya, e-mail: jgalaz@gmail.com; Wah Chiu,

18 e-mail: wahc@stanford.edu 


\section{ABSTRACT}

Huntington disease (HD) is a neurodegenerative trinucleotide repeat disorder

24 caused by an expanded poly-glutamine (polyQ) tract in the mutant huntingtin (mHTT)

25 protein. The formation and topology of filamentous mHTT inclusions in the brain

26 (hallmarks of HD implicated in neurotoxicity) remain elusive. Using cryo-electron

27 tomography and subtomogram averaging, here we show that mHTT exon 1 and polyQ-

28 only aggregates in vitro are structurally heterogenous and filamentous, similar to prior

29 observations with other methods. Yet, we find filaments in both types of aggregates under

$30 \sim 2 \mathrm{~nm}$ in width, thinner than previously reported, and regions forming large sheets. In

31 addition, our data show a prevalent subpopulation of filaments exhibiting a lumpy slab

32 morphology in both aggregates, supportive of the polyQ core model. This provides a basis

33 for future cryoET studies of various aggregated $\mathrm{mHTT}$ and polyQ constructs to improve

34 their structure-based modeling as well as their identification in cells without fusion tags. 


\section{INTRODUCTION}

Huntington disease (HD) is a neurodegenerative, fatal trinucleotide repeat disorder caused by a polyQ expansion in exon 1 of mutant huntingtin (mHTT) exceeding a pathogenic threshold of $Q>\sim 35^{1}$. Patients suffer from motor and cognitive impairments and despite our increased understanding of $\mathrm{HD}^{2}$ and promising clinical trials ${ }^{3}$, cures and preventive treatments remain elusive ${ }^{4}$. "mEx1") elicits HD phenotypes in cellular and animal models ${ }^{5-8}$, including primates 9 .

51 Furthermore, mEx1 inclusions in mouse and human brains ${ }^{10,11}$ are morphologically similar 52 to those in R6/2 and mEx1 knock-in mice ${ }^{12}$.

A polyQ expansion in different genes causes at least eight other disorders with a

54 similar pathogenic Q-repeat length threshold, irrespective of flanking motifs ${ }^{13,14}$, and 55 polyQ peptides as short as $Q=20$ are toxic when they contain a nuclear localization 56 signal ${ }^{15}$. Since structure often determines function ${ }^{16}$, as shown for mHTT toxic 57 aggregates ${ }^{17,18}$, an increased structural understanding of polyQ aggregates can help 58 uncover the mechanisms underlying their biogenesis, development, and cytotoxicity to 59 better model polyQ disorders. Both small mHTT oligomers and large inclusion bodies (IBs) can be neurotoxic ${ }^{19,20}$.

61 Filamentous aggregates of mEx1 constructs with various polyQ lengths (mEx1-Qn) have 62 been amply visualized with negative staining transmission electron microscopy (NS63 TEM $)^{21-23}$, a technique often limited to 2D projections and subject to metal stain and drying 64 artifacts. Two recent studies used cryo focused ion beam milling and electron tomography 65 (cryoFIB-ET) to visualize transfected mEx1-Q97 forming IBs within yeast ${ }^{24}$ and HeLa ${ }^{25}$ 
cells. However, a green fluorescence protein (GFP) fusion tag was used, which can alter mEx1 aggregation ${ }^{26}$.

Here, we used direct observation (without heavy metal stain or fusion tags) by cryo-electron tomography (cryoET) and subtomogram averaging (STA) ${ }^{27,28}$ to visualize vitrified filamentous mEx1-Q51 and Q51 (a peptide consisting of only glutamines) aggregates in vitro. We leveraged our initial observations of mEx1-Q51 filaments by cryoET ${ }^{29-31}$ and capitalized on recent algorithmic developments including compressed sensing for tomographic reconstruction ${ }^{32,33}$, convolutional neural networks for feature annotation $^{34}$, and automated fiducial-less tiltseries alignment and subtiltseries refinement for subtomogram averaging ${ }^{35}$ to resolve previously unattainable structures. Our study provides a three-dimensional (3D), nanometer-resolution structural description of untagged, vitrified mEx1 and Q-only aggregates, finding filaments that are thinner than previously observed, laminated sheets, and a predominant conformation exhibiting a lumpy slab morphology that supports the polyQ core model.

\section{RESULTS}

\section{Mutant huntingtin exon 1-Q51 filaments exhibit a large variation in width, narrow} branching angles, and lamination

We analyzed tomographic tiltseries of vitrified mEx1-Q51 (Figure 1a), collected as previously described (see Methods) ${ }^{29}$. Owing to the higher contrast and minimized missing wedge artifacts attainable with compressed sensing (CS) compared to standard weighted back projection ${ }^{32}$, we incorporated CS in our pipeline to reconstruct the tiltseries 
88 into tomograms (see Methods), which exhibited aggregated filamentous densities (Figure

89 1b,c). While CS might introduce artifacts at high resolution in the subnanometer range, it

90 has been demonstrated to produce faithful reconstructions at nanometer resolution ${ }^{36}$. The

91 most frequently observed widths from aggregates in six tomograms ranged between $\sim 5$

92 and $\sim 16 \mathrm{~nm}$, with the thinnest filaments exhibiting regions down to $\sim 2 \mathrm{~nm}$ thickness

93 (Figure 1d,e). On the other hand, the thickest filaments measured over $\sim 20 \mathrm{~nm}$ in width.

94 These measurements are not consistent with a cylindrical shape of a single radius, as

95 reported in recent cryoFIB-ET studies ${ }^{24,25}$. Rather, our observations are consistent with a

96 heterogeneous plethora of thin filaments, rectangular prisms, and even sheets of varying

97 size. We interpret the predominant species among our observed filaments as 3D

98 rectangular slabs, which could exhibit many different center-slice widths in between their

99 widest and narrowest dimensions when sliced computationally at slanted angles. Our

100 computational simulations of filamentous subtomograms using EMAN2 ${ }^{37}$ support this

101 model (Supplementary Figure S1). 
102 Figure 1. MEx1-Q51 filaments

103 exhibit a large variation in width

104 within and across filaments. (a)

105 Schematic of the mEx1-Q51

106 construct. (b) Slice parallel to xy $(\sim 1.7$

$107 \mathrm{~nm}$ thick) through a representative $4 \mathrm{x}$

108 down-sampled cryoET tomogram of

109 aggregated mEx1-Q51, reconstructed

110 with compressed sensing, lightly

111 filtered to enhance visualization, and

112 (c) corresponding semi-automated 3D

113 annotation. (d) Selected areas from

114 slices of large mEx1-Q51 aggregates

115 showing individual filaments, widely

116 varying in width, with the thinnest

117 filaments exhibiting regions down to

$118 \sim 2 \mathrm{~nm}$ width, indicated by the red

119 arrows. (e) Zoomed-in view of a xy

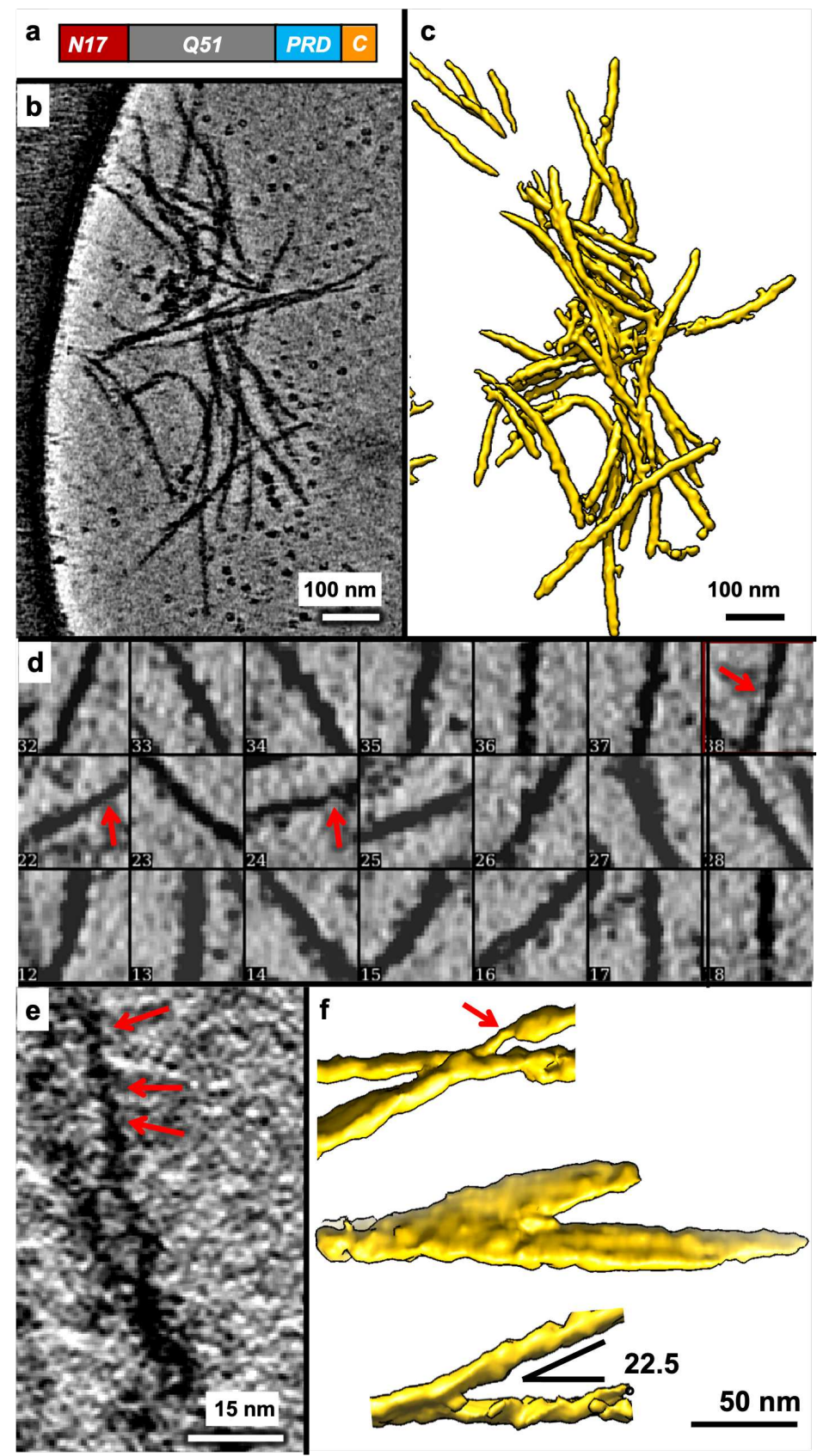

120 slice ( $0.4 \mathrm{~nm}$ thick) from a selected region of a tomogram without any down-sampling,

121 showcasing ultra-thin regions in mEx1-Q51 filaments. (f) Sections of annotated mEX1-

122 Q51 filamentous aggregates from cryoET tomograms showing relatively narrow

123 branching angles and an example of a thicker laminated sheet-like region (the annotation

124 example in the middle). 
We used semi-automated annotation based on neural networks ${ }^{34}$ to visualize in

127 3D what kind of objects yielded the extensive width variations detectable in 2D slices of

128 3D tomograms. Visualizing mEx1 filaments as 3D isosurfaces (Figure 1C;

129 Supplementary Figure S2) revealed filaments of different dimensions altogether,

130 including regions that appeared as sheets as thick as $\sim 50 \mathrm{~nm}$, estimated from the

131 annotations and from their persistence through 2D slices. The mEx1 filaments seemed to 132 predominantly branch out at angles varying from $\sim 10^{\circ}$ to $\sim 45^{\circ}$ (only sporadically larger),

133 with angles between $\sim 20^{\circ}$ to $\sim 25^{\circ}$ being most common (Figure 1f).

134 Subpopulation of mEx1-Q51 filament segments exhibits a lumpy, slab-shaped 135 morphology

136 Many filaments appeared to be lumpy both in 2D slices from 3D tomograms

137 (Figure 2a) as well as in in 3D annotations (Figure 2b), suggestive of potential periodicity.

138 Thus, we performed subtomogram averaging (STA) of filament segments, avoiding 139 obviously-laminated regions and thick bundles. The subtomogram average of mEx1-Q51

140 filament segments ( $\mathrm{n}=450$, from 6 tomograms) converged to a lumpy $\sim 7 \times 15 \mathrm{~nm}$ slab at

$141 \sim 3.5 \mathrm{~nm}$ resolution (Figure 2c). The Fourier transform of 2D re-projections of the average 142 did not reveal crisp layer lines, in agreement with previous studies suggesting that mEx1 143 filaments do not exhibit a canonical amyloid structure with parallel subunits stacked 144 helically in register ${ }^{22}$. Indeed, HD does not strictly fit among the diseases known as 145 amyloidoses ${ }^{38}$; nonetheless, the power spectra showed bright maxima off of the meridian, 146 at $\sim 11.7 \mathrm{~nm}$ (Supplementary Figure 4a), suggestive of potential periodicity for at least 147 relatively short stretches ( $\sim 65 \mathrm{~nm}$, the length included in the extracted subtomograms). 


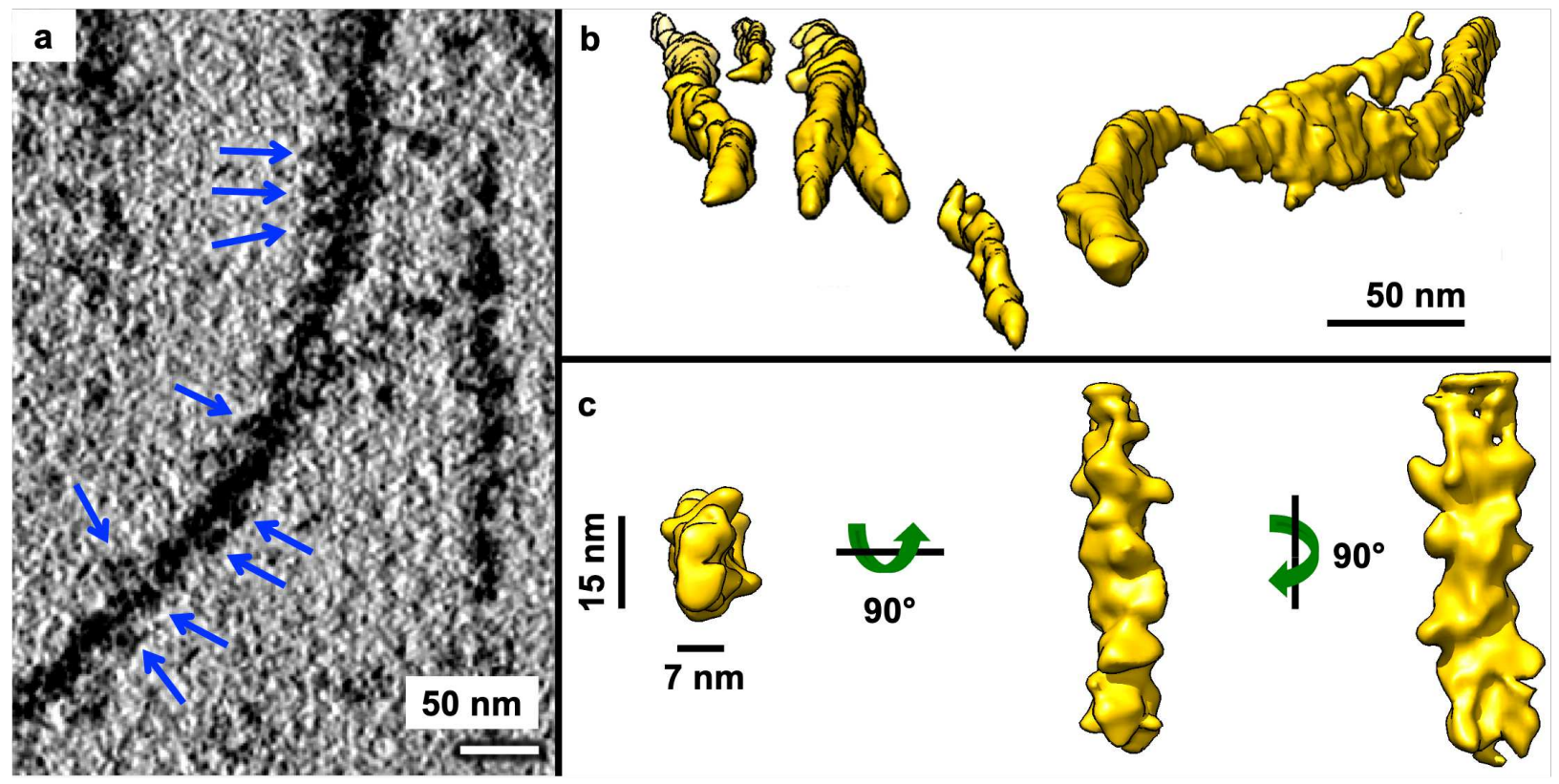

149 Figure 2. Aggregated mEx1-Q51 exhibits lumpy, slab-shaped filaments. (a) Pseudo-

150 periodic pattern of repeating lumps (blue arrows) along the length of an mEx1-Q51

151 filament as seen in an xy slice (4.4 $\AA$ thick) from a tomogram of aggregated mEx1-Q51.

152 (b) Selected regions from semi-automated neural network annotations showing lumpy

153 filaments of various widths, including sheet-like regions (middle region of right-most

154 example). (c) Subtomogram average of a subpopulation of filament segments exhibiting 155 a lumpy $7 \times 15 \mathrm{~nm}$ slab-shaped morphology.

Lumpy, slab-shaped Q51-only filaments also exhibit lamination

Since an expanded polyQ tract is the common culprit of all polyglutamine diseases,

159 we performed the same analyses for a Q51-only peptide (Figure 3a) as reported above

160 for mEx1-Q51. We found that Q51 also forms aggregates (Figure 3b, Supplementary

161 Figure 3) exhibiting lumpy filaments (Figure 3c) of varying widths (Figure 3d,e), with 162 regions as thin as $\sim 2 \mathrm{~nm}$. 


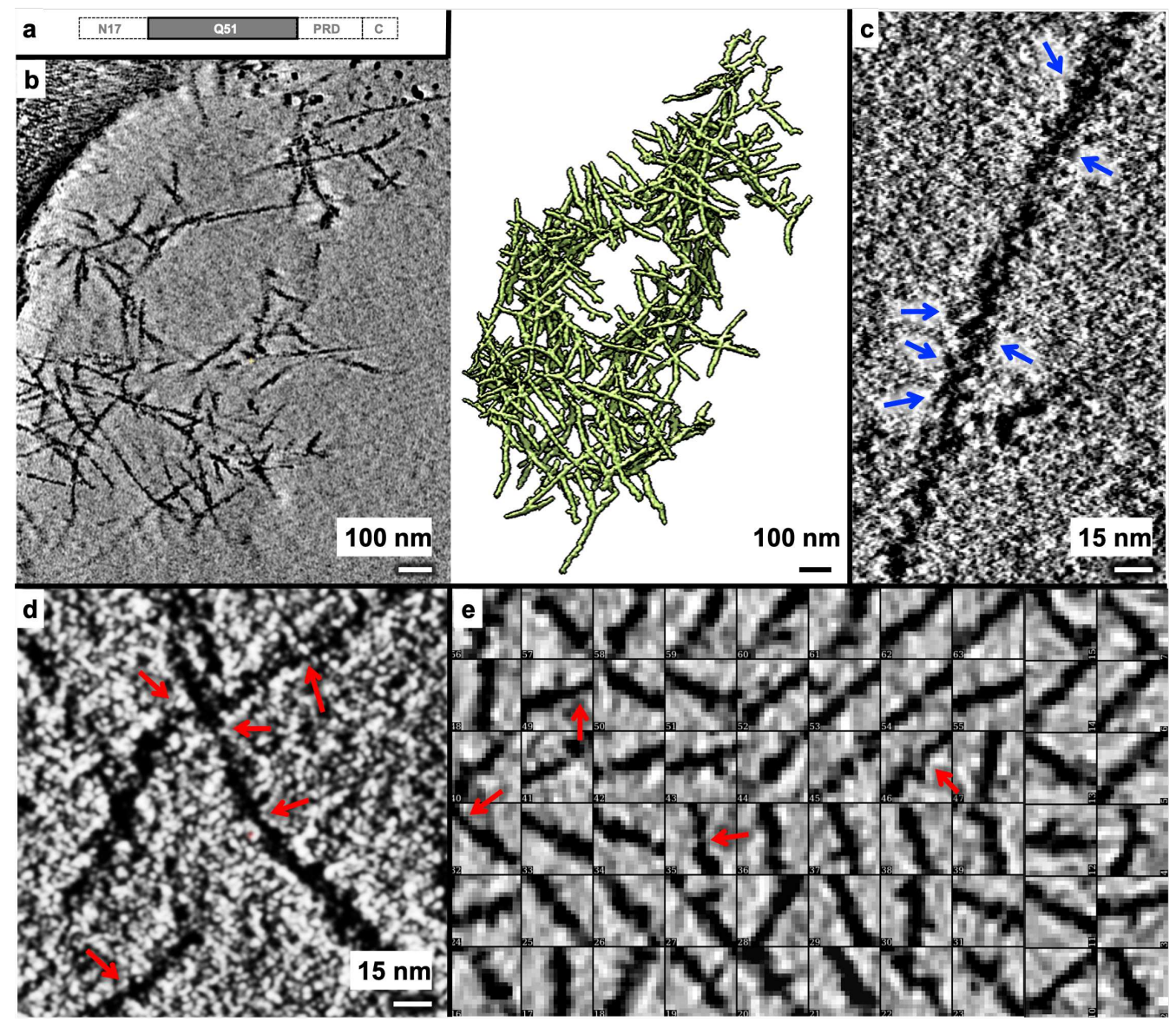

164 Figure 3. Lumpy Q51 filaments exhibit a large range of widths. (a) Schematic of the

165 Q51 construct, lacking all mEx1 domains except for the polyQ tract. (b) Slice parallel to

166 the xy plane ( $\sim 2.1 \mathrm{~nm}$ thick) through a representative $4 \mathrm{x}$ down-sampled cryoET tomogram

167 of aggregated Q51 reconstructed with compressed sensing (left) and corresponding 3D

168 annotation (right). Zoomed-in views of xy slices ( $\sim .5 \mathrm{~nm}$ thick) from selected regions of

169 the tomogram shown in A but without any down-sampling, exhibiting (c) a pseudo-

170 periodic pattern of repeating lumps along the length of a Q51 filament (blue arrows), and

171 (d) regions in thin filaments that are as thin as $\sim 2 \mathrm{~nm}$ in width (red arrows). (e) Examples

172 of 2D xy slices through representative 3D subtomograms of Q51 filaments showing a

173 wide variation in width, including super-thin regions $\sim 2 \mathrm{~nm}$ in width (red arrows). 
174 Figure 4. Aggregated Q51 exhibits lamination sheets and predominantly lumpy,

a

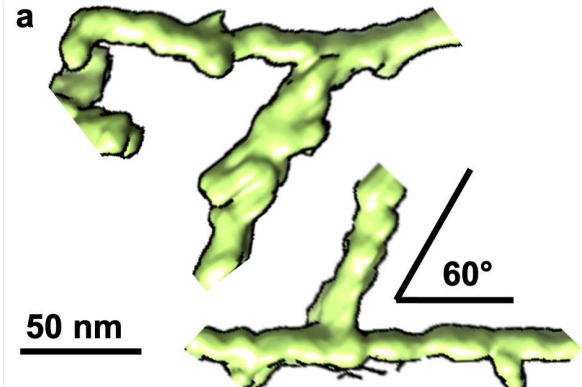

b

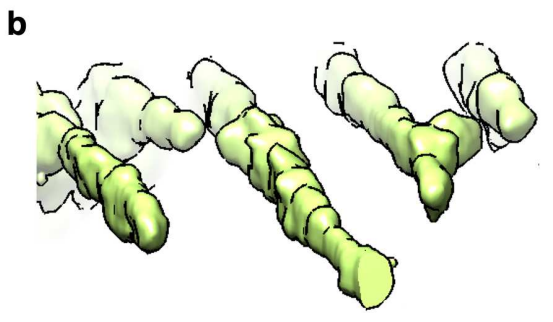

$50 \mathrm{~nm}$

c

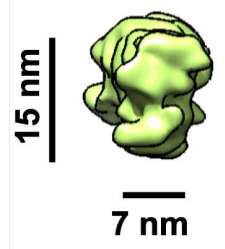

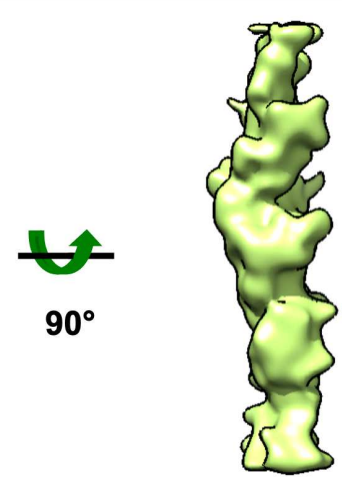
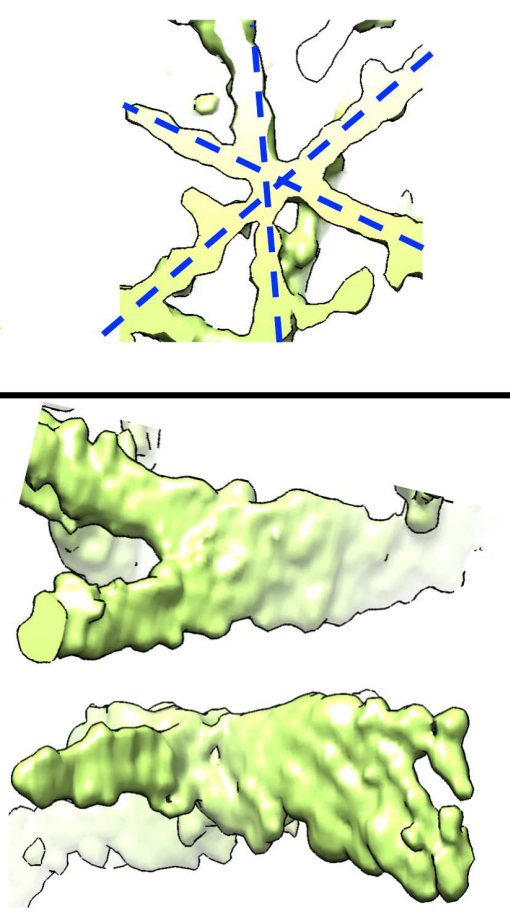

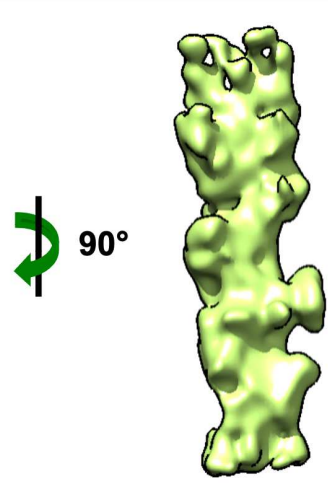

slab-shaped filaments. (a)

Representative sections of annotated Q51 filamentous aggregates from cryoET tomograms showing their most common branching/crossover angle $\left(\sim 60^{\circ}\right)$, often in an asterisklike pattern, and (b) thicker regions (right) akin to lamination, onside thinner ones (left). (c) Subtomogram average of a subpopulation of filament segments exhibiting a lumpy $7 \times 15 \mathrm{~nm}$ slab-shaped 190 morphology.

193 mEx1-Q51 filaments, with $\sim 60^{\circ}$ being the most common angle (Figure 4a). Furthermore,

194 Q51 filaments exhibited larger lamination sheets than those of mEx1-Q51 of up to 60+

$195 \mathrm{~nm}$ in width (Figure 4b). The subtomogram average of non-laminated filament segments 196 ( $n=493$, from 6 tomograms) converged to $\sim 3.2 \mathrm{~nm}$ resolution and also revealed a lumpy 
$197 \sim 7 \times 15 \mathrm{~nm}$ slab (Figure 4c), with a crossover length of $\sim 11.2 \mathrm{~nm}$ according to the power

198 spectrum of its projections (Supplementary Figure 4b), all strikingly similar results to 199 those obtained for mEx1-Q51.

\section{DISCUSSION}

In one of the earliest reports visualizing mEx1-Q51 filamentous aggregates by NS-

TEM, filaments digested with factor Xa or trypsin, which removes mEx1's N17 domain critical to $\mathrm{mHTT}$ localization and function ${ }^{39}$, were reported to have a diameter of $\sim 7.7-12$ $\mathrm{nm}$ from 2-dimensional (2D) images ${ }^{40}$. These were occasionally referred to as ribbons, and other 2D NS-TEM observations have reported similar filaments with a 10-12 nm diameter, which may associate laterally ${ }^{22}$. However, apparent lateral associations in 2D NS-TEM observations may arise from the compression of all densities into a single layer

209 due to dehydration. In contrast, the vitrified filaments seen in our 3-dimensional (3D) 210 tomograms varied much more in width within and across filaments, similar to those 211 reported in studies visualizing aggregated Q-only peptides with NS-TEM ${ }^{41,42}$, which also

212 detected wide ribbons and thin filaments under different incubation temperatures and 213 using a freeze-concentration method involving cycles of freezing and thawing. Here, our 214 observations did not suffer from NS-TEM artifacts and were conducted at $30^{\circ} \mathrm{C}$, without 215 special temperature manipulations.

While other amyloidogenic filaments have been amply studied using cryo electron 217 microscopy (cryoEM) ${ }^{43-50}$, cryoEM studies of $\mathrm{mHTT}$ and polyQ-containing aggregates

218 have been extremely scant in comparison, likely due to the extensive conformational 219 heterogeneity of these specimens ${ }^{51}$, which limits the applicability of bulk techniques (e.g., 
220 Circular Dichroism) and calls for the increasing application of single molecule 221 techniques ${ }^{52}$. Single molecule techniques such as Atomic Force Microscopy (AFM) and 222 various modalities of electron microscopy (EM) can observe individual components in 223 aggregates (molecules, oligomers, filaments). For EM-related methods, these 224 components can be classified prior to averaging. Of note, cryoET is the most suitable 225 method to investigate the overall structure of relatively large and thick, hydrated samples 226 exhibiting extensive conformational and compositional heterogeneity as it avoids 227 adsorption of the specimen onto 2D surfaces and the confounding effect of potentially 228 overlapping densities from different components, as in 2D projections produced by single 229 particle cryoEM.

230 In two recent cryoFIB-ET studies, mEx1-Q97-GFP filaments were observed and 231 annotated in transfected cellular systems but were not averaged. Rather, they were either 232 modeled as cylinders with an 8-nm diameter for template-based annotation ${ }^{25}$ or were 233 segmented as $16 \mathrm{~nm}$ filaments ${ }^{24}$, surprisingly twice as thick in the latter study than in the 234 former, perhaps owing to differences in the non-native expression systems used or to the 235 confounding presence of GFP fusion tags. Indeed, there can be caveats to using fusions 236 to fluorescent proteins as tags, from impairing the viability and growth of cells via toxic 237 effects from tag aggregation, excitation, or photoactivation, to changing the structure, 238 function, and cellular localization of the tagged protein ${ }^{53}$. Template-based approaches 239 have been successfully applied to annotate more regularly-shaped biological 240 filaments ${ }^{54,55}$; however, our data here suggest that the use of a cylindrical template is not 241 an adequate approach to annotate widely heterogeneous $\mathrm{mEx} 1$ and polyQ aggregates 242 with filamentous densities of varying dimensions. Indeed, when identifying features in 
243 tomograms, template matching can be biased ${ }^{56}$ and manual human annotation is

244 subjective and therefore often uncertain and inconsistent ${ }^{57}$. On the other hand, here we

245 used template-free, semi-automated annotation based on machine learning since it can

246 ameliorate these issues by minimizing human input and the use of a priori constraints

247 inherent in templates ${ }^{34}$.

248 A recent atomic force microscopy (AFM) study on mEx1-Q49 aggregation 249 suggested that nucleated branching from filaments, rather than lateral associations 250 among them, leads to large bundles ${ }^{58}$. However, branching does not explain how the 251 thinner ( $<2 \mathrm{~nm}$ thick) filaments that we observe here would assemble into thicker slabs 252 and sheets without associating laterally or growing transversally to the main filament axis. 253 Rather, our results suggest that preformed thin filaments can associate laterally and/or 254 that growing filaments can expand transversally in addition to longitudinally, akin to the 255 lamination observed for $A \beta^{59}$, for both mEx1-Q51 and Q51.

257 specimens that are often absorbed and dried onto a 2D substrate, an earlier AFM study 258 of aggregated Q44 peptide detected regions in filament tips with a "height" (the size of 259 the specimen in the direction perpendicular to the adsorption surface) as thin as $\sim 5 \mathrm{~nm}^{60}$. 260 This and the thinness of some of the filament regions we observed here (as thin as $\sim 2$ $261 \mathrm{~nm}$ ) seem to disagree with the minimum width of $\sim 7-8 \mathrm{~nm}$ proposed for polyQ filaments 262 from various Qn constructs in a prior NMR study that also presented NS-TEM images ${ }^{61}$. 263 However, the latter study reports filament widths for a Q54 peptide from NS-STEM 264 images from $\sim 7-8 \mathrm{~nm}$ up to $\sim 16 \mathrm{~nm}$, in striking agreement with the short and long sides 
265 of the slab-shaped model we propose here as the predominant morphology for filaments 266 formed by both mEx1-Q51 and Q51.

267 The morphological characteristics deviating from thin cylindrical shapes to form 268 lumpy slabs and sheets may serve as a structural hallmark to identify untagged $\mathrm{mHTT}$ 269 aggregates in cells. Furthermore, the more frequent and wider-angle branching of Q-only 270 filaments compared to $\mathrm{mEx} 1$ is consistent with our prior $2 \mathrm{D}$ cryoEM observations ${ }^{31}$, 271 suggesting that the N17 domain promotes inter-filament bundling. Conversely, the 272 occurrence of branching might be primarily polyQ-driven. Indeed, our 3D observations 273 here, which are free from fusion tags, stains, dehydration, flattening, and crystallization 274 artifacts, provide a transforming complement and clarification to previous studies by NS275 TEM and AFM, as well as light microscopy ${ }^{62,63}$, which visualized $m E x 1$ filamentous 276 aggregates at a coarser level: features often described as globules or oligomers or thick 277 filaments actually correspond to bundles of many interwoven thinner filamentous 278 densities when viewed by cryoET.

279 The fact that the predominant populations for both $\mathrm{mEx} 1$ and Q-only filaments 280 exhibit a similar lumpy slab shape and distance between putative crossovers as revealed 281 by subtomogram averaging suggests that the morphology of their core is dictated by and 282 primarily comprised of the polyQ tract, and that the flanking domains in $\mathrm{mEx} 1$ are largely 283 exposed at the filament surface, allowing them to modulate inter-filament aggregation. 284 This interpretation agrees with previous nuclear magnetic resonance (NMR) studies on 285 non-pathogenic ${ }^{64}$ and pathogenic ${ }^{65,66} \mathrm{mEx} 1$ variants that propose the existence a dense 286 polyQ core. 
In one of the latest studies supporting the polyQ-core model ${ }^{67}$, the authors 288 observed mEx1-Q44 filaments formed at two different temperatures by 2D NS-TEM 289 images (presented in the supplement). The widths reported for these filaments were $\sim 6.5$ $290 \mathrm{~nm}$ and $\sim 15.2 \mathrm{~nm}$, in striking agreement with the dimensions of our slab-shaped 291 subtomogram averages of filament segments from 3D cryoET tomograms of vitrified $292 \mathrm{mEX}-\mathrm{Q} 51$ and Q51. While their hypothesis that the thicker $\sim 15.2 \mathrm{~nm}$ filaments must arise 293 from two interwinding protofilaments $\sim 6.5 \mathrm{~nm}$ thick seems to be compatible with our 294 observations here, their model proposing that the flanking domains mediate such 295 interwinding does not explain our observation that polyQ-only filaments also yield a 296 dominant subpopulation with the same $\sim 7 \times 15 \mathrm{~nm}$ slab morphology, which could also 297 correspond to two interwoven protofilaments without flanking domains to bind them. If, 298 indeed, the mEx1-Q51 and Q51-only predominant subpopulations of $\sim 7 \times 15 \mathrm{~nm}$ filaments 299 are composed of two thinner interwinding protofilaments, our data suggest that they might 300 be bound primarily via polyQ-polyQ interactions.

Our observations here warrant further cryoET experiments with much larger 302 datasets of aggregation-competent mEx1 and polyQ-only constructs devoid of 303 solubilization and purification tags, as even these can cause modest alterations in 304 aggregation kinetics ${ }^{68,69}$. Datasets at higher magnification and contrast, using state-of305 the-art instrumentation, could test whether there exist filament species even thinner than 306 the $\sim 2 \mathrm{~nm}$ regions we observed here, as could probe the effects of increasing polyQ 307 length on the 3D morphologies of vitrified filamentous aggregates. Assessing the effects 308 of post-translational modifications (PTMs) on filament and overall aggregate structure 309 with cryoET might be of particular significance, as some PTMs modulate aggregation with 
310 neuroprotective effects ${ }^{70}$. Finally, sonication concomitant with trypsin digestion of $\mathrm{mEx} 1$

311 filaments might yield a homogenous-enough population of the polyQ core that may be 312 more amenable to higher-resolution cryoEM/ET studies.

\section{METHODS}

315 In vitro mEx1-Q51 and Q51 peptide aggregation assays and cryoET sample preparation

We used mutant huntingtin (mHTT) exon 1 with 51 glutamine repeats (mEx1-Q51) and a polyQ-only peptide with 51 repeats (Q51), each of them fused to a TEV cleavage

319 sequence and a GST tag, as previously described ${ }^{31}$. Aggregation was initiated separately 320 at a concentration of $6 \mu \mathrm{M}$ for each construct in vitro by addition of AcTEV ${ }^{\mathrm{TM}}$ protease 321 (Invitrogen), as previously described for mEx1-Q51 ${ }^{29}$. The samples were incubated at 30 $322{ }^{\circ} \mathrm{C}$ before vitrification. Aliquots of $2.5 \mu \mathrm{m}$ were separately applied to 200 -mesh holey 323 carbon Quantifoil copper grids (previously washed with acetone, and rinsed in PBS)

324 between 4 and $6 \mathrm{~h}$ post-initiation of aggregation. The grids were plunge-frozen in a liquid 325 ethane bath kept at liquid nitrogen temperature using a Vitrobot Mark III (FEI Instruments).

\section{Tiltseries collection}

We collected six tiltseries of the Q51 peptide using SerialEM software ${ }^{71}$ on a 328 JEM2100 electron microscope operated at $200 \mathrm{kV}$ from $-60^{\circ}$ to $60^{\circ}$ in $2^{\circ}$ increments, at 6

$329 \mu \mathrm{m}$ target underfocus, $5.29 \AA /$ pixel sampling size, with a cumulative dose of $\sim 80 \mathrm{e} / \AA^{2}$. We 330 also reanalyzed a previous dataset comprised of 20 tiltseries of mEx1-Q51 + TRiC, 
331 collected similarly to the Q51 peptide dataset, as previously described ${ }^{29}$, with a slightly

332 finer sampling size of $4.4 \AA /$ pixel.

\section{Tomographic reconstruction}

All mEx1-Q51 and Q51 tilt series were binned by 2x and initially aligned and reconstructed into tomograms with $\mathrm{IMOD}^{72}$. Images with artifacts (grid bars in the field of

336 view blocking large regions of the specimen, evident large drift, obvious radiation

337 damage, etc.) were manually removed prior to tiltseries alignment and tomographic

338 reconstruction with weighted back projection. After assessing sample thickness, the

339 tiltseries were reconstructed again into tomograms using compressed sensing (CS) as

340 implemented in ICON-GPU ${ }^{32,33}$ to improve contrast. Of note, CS also partially restores

341 information that is lacking due to the missing wedge artifact inherent in all conventional

342 single-axis limited-angle tomography experiments, such as conventional cryoE $T^{73}$. The

343 tiltseries were aligned and reconstructed yet a third time for subtomogram averaging

344 purposes (as described below), using a new pipeline for cryoET in EMAN2 ${ }^{35}$ that performs

345 sub-tiltseries refinement, akin to prior hybrid methods combining concepts from single

346 particle analysis cryoEM and subtomogram averaging ${ }^{74-76}$. We processed the mEx1-Q51

347 and Q51 datasets separately in virtually identical ways.

348 Tomogram annotation

349 Since the ultimate goal of the new EMAN2 cryoET pipeline ${ }^{35}$ is to perform 350 subtiltseries refinement for subtomogram averaging, tomogram quality only needs to be 351 sufficient to allow for particle identification. Indeed, in EMAN2 not as many parameters 352 are refined during tomographic reconstruction as compared to IMOD, often resulting in 353 lower-quality tomograms. For this reason, we performed all tomographic annotations on 
354 better-quality tomograms aligned with IMOD and reconstructed with $\mathrm{CS}$, as described

355 above. MEx1-Q51 and Q51 annotations were carried out on binned-by-4 tomograms 356 using EMAN2's neural network semi-automated annotation tools ${ }^{34}$, except that $\sim 2-3 x$ as 357 many references as the 10 recommended were segmented to seed annotation, and $\sim 2-$

$3583 \mathrm{x}$ as many negative samples as the 100 recommended were selected to minimize false 359 positives. We initially performed annotation of all mEx1-Q51 and Q51 tomograms by 360 applying the convolutional neural network from the best tomogram to all the rest, 361 separately for each specimen. However, false positives (such as annotating the carbon362 hole edge and/or gold fiducials) were reduced further when we generated a neural 363 network specific for each mEx1-Q51 and Q51 tomogram.

\section{Fibril width range measurements}

365 In all limited-angle tomography experiments (when you cannot tilt through the 366 entire full range from $0^{\circ}$ to $180^{\circ}$ or $-90^{\circ}$ to $+90^{\circ}$ to collect a full set of projections around 367 the object of interest), the missing wedge artifact worsens the resolution of raw 368 tomograms along the $Z$-axis compared to that in the $X$ and $Y$ directions, often giving the 369 appearance of elongation of features along the axis with lowest resolution. Therefore,

370 filament widths cannot be accurately measured in 3D from raw tomograms nor their 371 corresponding annotations in arbitrary orientations. The most conservative 372 measurements in the absence of averaging should be performed on slices along the Z373 axis of reconstructed tomograms (i.e., on sections parallel to the XY plane) since features 374 are much less well-resolved in the $X Z$ and $Y Z$ planes. Here, we boxed out filament 375 segments for STA (below) and manually measured the thinnest and thickest parts of 
376 segments $(\mathrm{N} \sim 100)$ from the central $\mathrm{XY}$ slice of the corresponding subtomogram. The

377 mEx1-Q51 and Q51 data were separately processed in identical ways.

\section{Initial model generation for subtomogram averaging (STA)}

To carry out sub-tiltseries refinement, the new EMAN2 cryoET pipeline ${ }^{35}$ requires

that all steps (from initial tomographic reconstruction) be performed in EMAN2. However,

381 as explained above, whole-tiltseries alignment with IMOD is often superior in quality,

382 given its refinement of more reconstruction parameters, and reconstruction with ICON-

383 GPU can yield higher-contrast tomograms with minimized missing wedge artifacts.

384 Therefore, to generate an initial model, we manually extracted filament segments without

385 much overlap from the best tomogram for each specimen $(n=97$ for $m E x 1-Q 51 ; n=135$

386 for Q51) avoiding branching points and regions of dense bundling or obvious lamination.

387 Then, we aligned these subtomograms to a cylindrical reference with a soft edge and 388 computed the average using the legacy tools for STA in EMAN2 ${ }^{77}$. This average of 389 vertically-aligned filaments was then refined constraining the angular search in altitude to 390 only allow for slightly-tilted orientations (since all particles were already pre-aligned to a 391 cylinder) and flips of $180^{\circ}$ in altitude (the other two Euler angles were completely 392 unconstrained). Alignment converged in $\sim 4-5$ iterations for both datasets. We used these 393 preliminary averages as initial models for subsequent unconstrained gold-standard 394 subtomogram averaging of $m E x 1-Q 51$ and Q51 with sub-tiltseries refinement in the new 395 EMAN2 pipeline.

\section{Subtomogram averaging}

Since the reconstruction geometry is different for tomograms produced with

398 different software packages, we had to pick subtomograms of filament segments (with < 
$\sim 50 \%$ overlap) manually from scratch ( $n=450$ for mEx1-Q51; $n=493$ for Q51) in EMAN2reconstructed tomograms. Gold-standard refinements seeded with the initial models

401 described above converged in $\sim 4-5$ iteration and $\sim 60 \%$ of the best-correlating particles 402 were kept in the final average for each dataset. The subtiltseries refinement step alone 403 improved the resolution drastically by $\sim 10 \AA$ or more for both datasets, yielding averages 404 at $\sim 3.5 \mathrm{~nm}$ and $\sim 3.2 \mathrm{~nm}$ resolution for mEx1-Q51 and Q51, respectively, according to the 405 gold-standard FSC=0.143 criterion.

\section{Visualization}

Tomographic slices were visualized with either EMAN2 ${ }^{37}$ or IMOD $^{72}$. All isosurfaces were visualized with UCSF Chimera ${ }^{78}$.

\section{STATISTICS AND REPRODUCIBILITY}

We collected six tomograms of each mEX1-Q51 and Q51 aggregates, and boxed

412 out 450 and 493 subtomograms of filament segments from each set, respectively, used

413 for filament width measurements and subtomogram averaging analyses, which are 414 thoroughly described in the Methods.

\section{DATA AVAILABILITY AND ACCESSION NUMBERS}

The raw data can be made accessible upon request. The Electron Microscopy

418 Data Bank accession numbers for the structures reported in this paper are as follows:

419 mEx1-Q51 subtomogram average, EMD-21248; Q51 subtomogram average, EMD42021253. 


\section{1}

422

423

424

425

426

\section{7}

428

429

\section{1}

432

433

\section{4}

435

\section{6}

437

438

439

440

\section{AUTHOR CONTRIBUTIONS}

All authors planned and designed experiments. K.S. generated and purified the mEx1-Q51 and Q51 constructs. S.H.S. collected the cryoET data. J.G.G.M. performed all data processing and analyses, prepared all figures, and wrote the manuscript with feedback from all authors.

\section{ACKNOWLEDGEMENTS AND FUNDING}

This research has been supported by grants from the National Institutes of Health, USA, No. NS092525 to J.F. and W.C., and No. P41GM103832 to W.C.

\section{CONFLICTS OF INTEREST DECLARATIONS}

The authors declare no conflicts of interest.

\section{REPORTING SUMMARY}

Further information on research design is available in the Nature Research Reporting Summary linked to this article. 

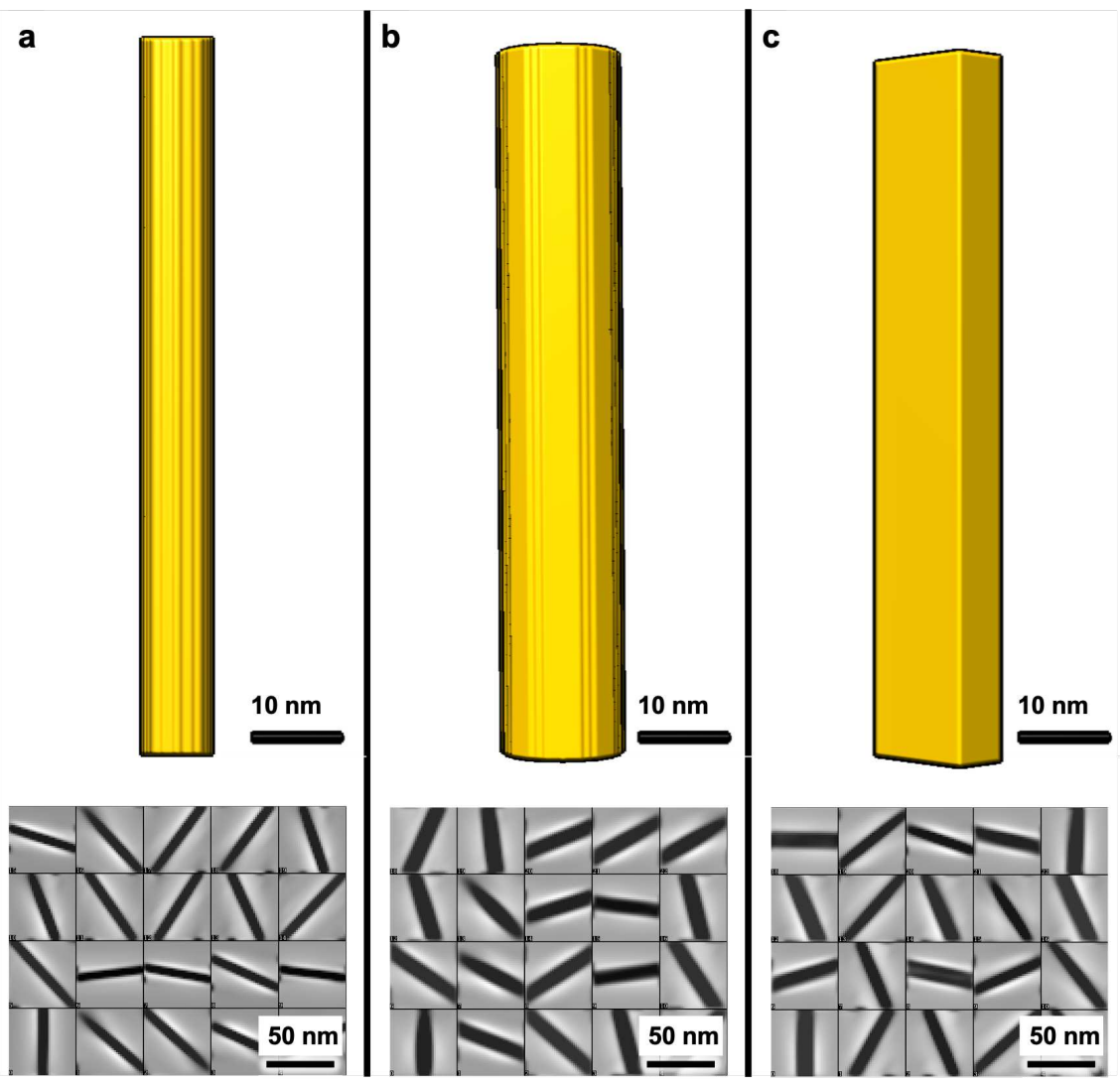

Supplementary Figure S1. Slab-shaped filaments are more consistent with 444 observations of variable width in central $Z$ cross-sections (in the $X Y$ plane, unaffected by the missing wedge artifact) than cylindrical filaments. Simulated

446 model and corresponding central $Z$ cross-sections of simulated subtomograms for a

447 cylinder (a) $7 \mathrm{~nm}$ or (b) $15 \mathrm{~nm}$ in diameter, and (c) a rectangular slab with narrow and 448 wide sides measuring 7 and $15 \mathrm{~nm}$, respectively. 


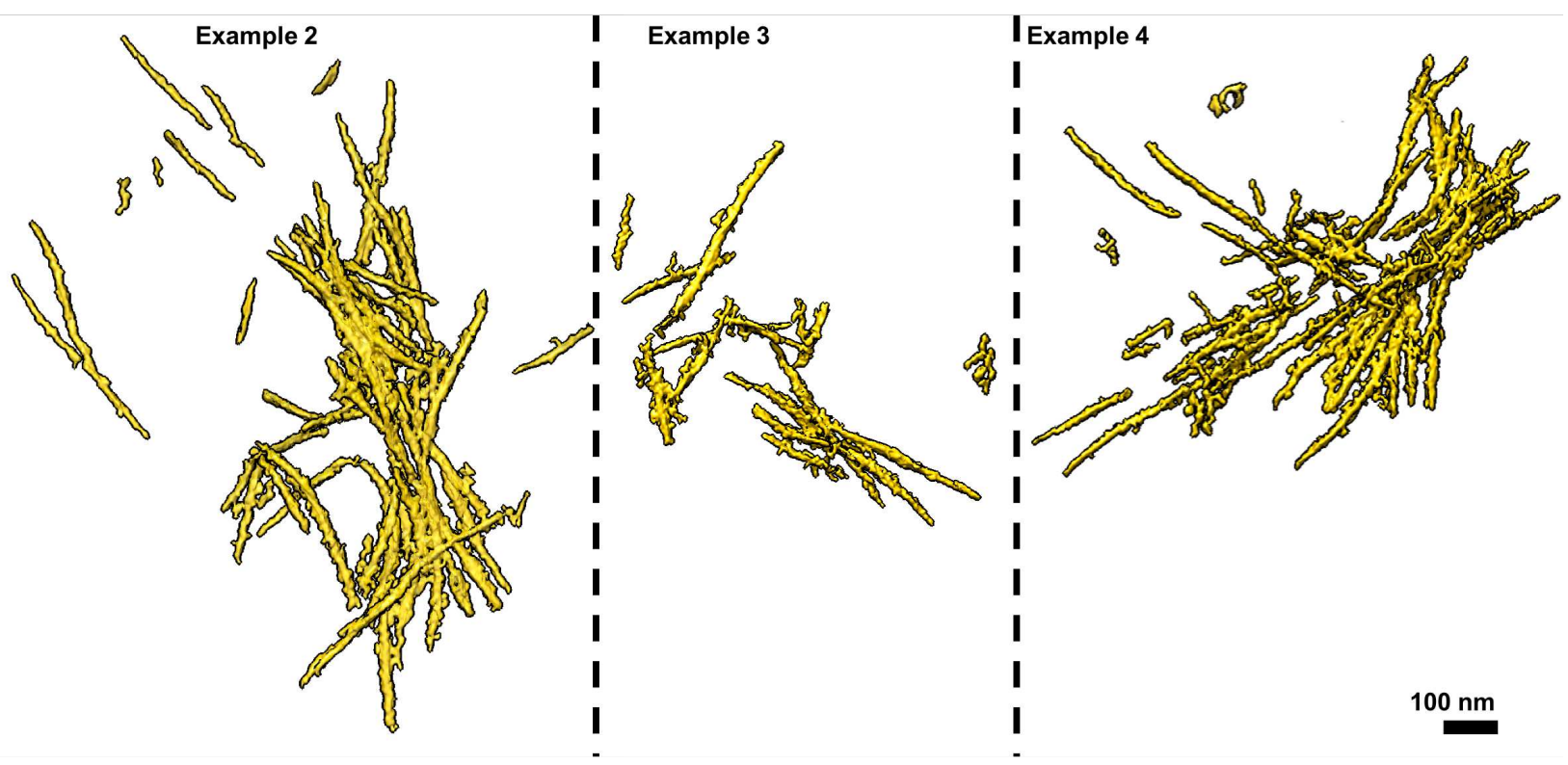

451 Supplementary Figure S2. Additional examples of mEx1-Q51 filamentous aggregates.

452
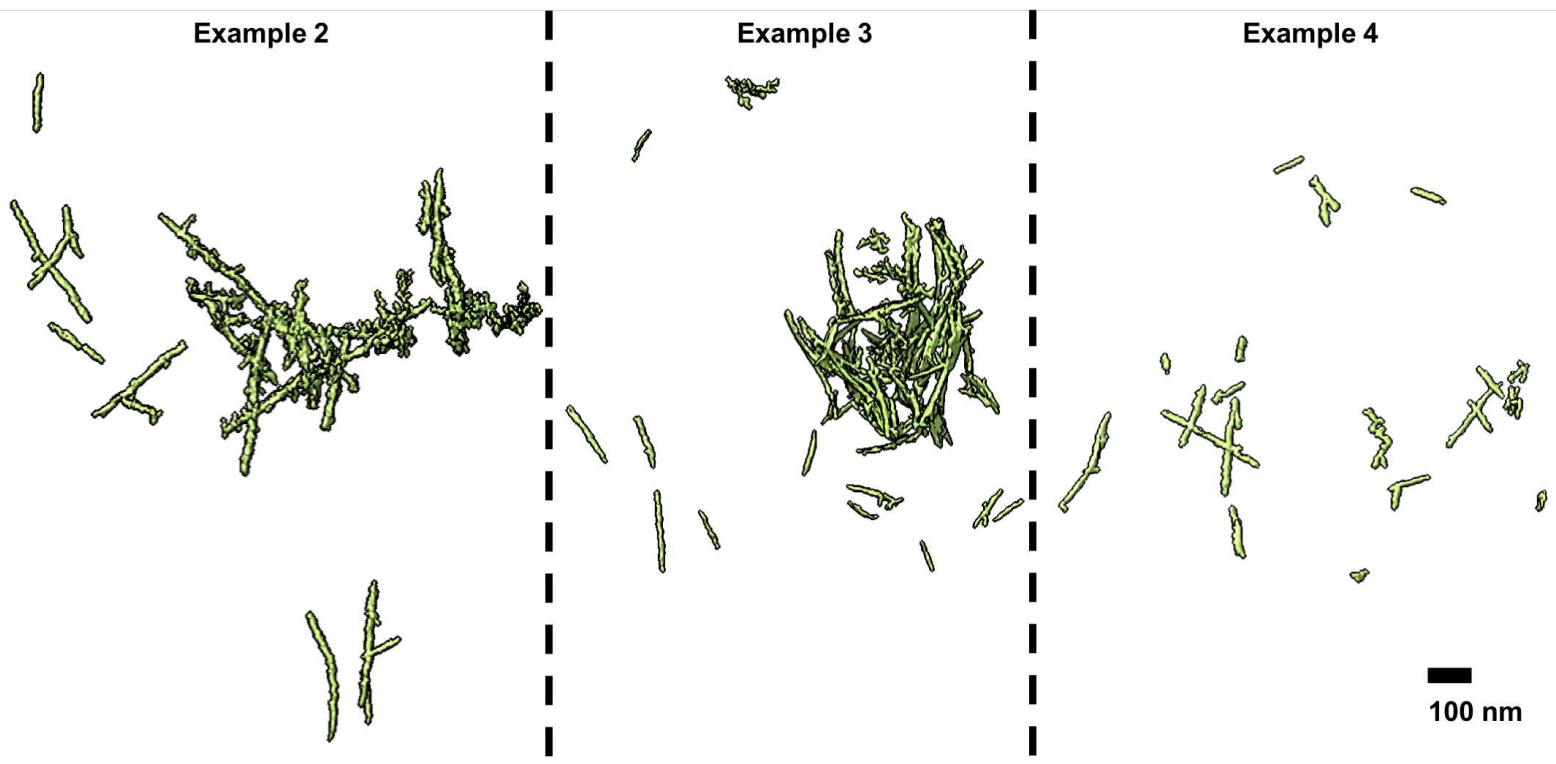

454 Supplementary Figure S3. Additional examples of Q51 filamentous aggregates. 


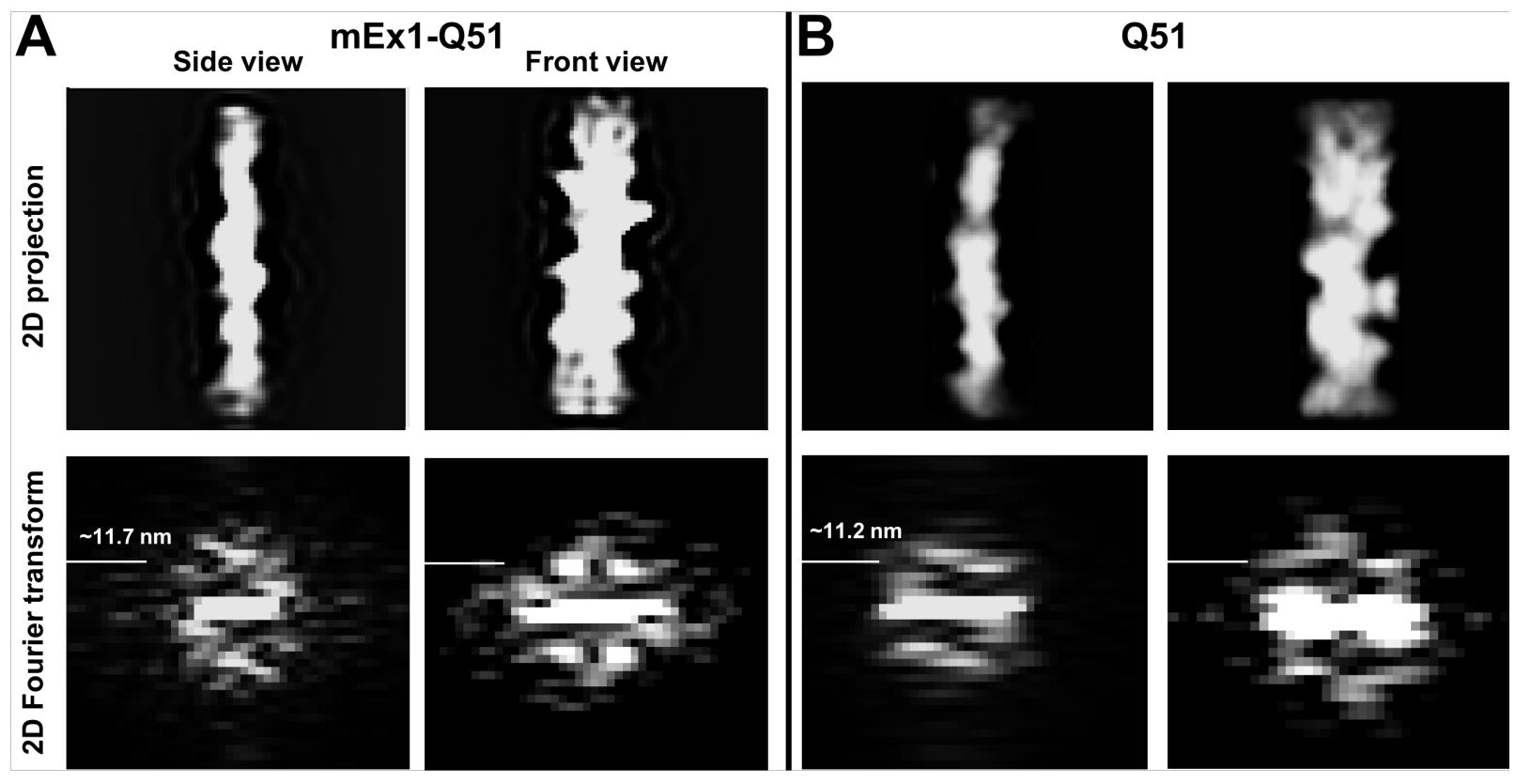

456 Supplementary Figure S4. Power spectrum of orthogonal side (left) and face-on (right)

457 projections from the subtomogram average of (a) mEx1-Q51 (Figure 2C) and (b) Q51 458 (Figure 4C) filaments. 


\section{REFERENCES}

4621 MacDonald, M. E. et al. A novel gene containing a trinucleotide repeat that is 463 expanded and unstable on Huntington's disease chromosomes. Cell 72, 971-983 464 (1993).

4652 Testa, C. M. \& Jankovic, J. Huntington disease: A quarter century of progress since 466 the gene discovery. J Neurol Sci 396, 52-68, doi:10.1016/j.jns.2018.09.022 (2019).

4673 Tabrizi, S. J., Ghosh, R. \& Leavitt, B. R. Huntingtin Lowering Strategies for Disease 468 Modification in Huntington's Disease. Neuron 101, 801-819, 469 doi:10.1016/j.neuron.2019.01.039 (2019).

4704 Wild, E. J. \& Tabrizi, S. J. Therapies targeting DNA and RNA in Huntington's 471 disease. Lancet Neurol 16, 837-847, doi:10.1016/S1474-4422(17)30280-6 (2017).

4725 Mangiarini, L. et al. Exon 1 of the HD gene with an expanded CAG repeat is 473 sufficient to cause a progressive neurological phenotype in transgenic mice. Cell 474 87, 493-506, doi:10.1016/s0092-8674(00)81369-0 (1996).

4756 von Hörsten, S. et al. Transgenic rat model of Huntington's disease. Hum Mol $476 \quad$ Genet 12, 617-624, doi:10.1093/hmg/ddg075 (2003).

4777 Wang, L. \& Sigworth, F. J. Cryo-EM and single particles. Physiology 21, 13-18 $478 \quad$ (2006).

4798 Wang, H. et al. Suppression of polyglutamine-induced toxicity in cell and animal 480 models of Huntington's disease by ubiquilin. Hum Mol Genet 15, 1025-1041, $481 \quad$ doi:10.1093/hmg/ddl017 (2006).

4829 Yang, S.-H. et al. Towards a transgenic model of Huntington's disease in a non483 human primate. Nature 453, 921-924, doi:10.1038/nature06975 (2008). 
48410 Davies, S. W. et al. Formation of neuronal intranuclear inclusions underlies the neurological dysfunction in mice transgenic for the HD mutation. Cell 90, 537-548, doi:10.1016/s0092-8674(00)80513-9 (1997).

48711 DiFiglia, M. et al. Aggregation of huntingtin in neuronal intranuclear inclusions and dystrophic neurites in brain. Science 277, 1990-1993, doi:10.1126/science.277.5334.1990 (1997).

49012 Sathasivam, K. et al. Identical oligomeric and fibrillar structures captured from the brains of R6/2 and knock-in mouse models of Huntington's disease. Hum Mol Genet 19, 65-78, doi:10.1093/hmg/ddp467 (2010).

49313 Gatchel, J. R. \& Zoghbi, H. Y. Diseases of unstable repeat expansion: mechanisms 494 and common principles. Nat Rev Genet 6, 743-755, doi:10.1038/nrg1691 (2005).

49514 Fan, H.-C. et al. Polyglutamine (PolyQ) diseases: genetics to treatments. Cell $496 \quad$ Transplant 23, 441-458, doi:10.3727/096368914X678454 (2014).

49715 Yang, W., Dunlap, J. R., Andrews, R. B. \& Wetzel, R. Aggregated polyglutamine 498 peptides delivered to nuclei are toxic to mammalian cells. Hum Mol Genet 11, 499 2905-2917, doi:10.1093/hmg/11.23.2905 (2002).

50016 Redfern, O. C., Dessailly, B. \& Orengo, C. A. Exploring the structure and function $501 \quad$ paradigm. Current opinion in structural biology 18, 394-402 (2008).

50217 Nekooki-Machida, Y. et al. Distinct conformations of in vitro and in vivo amyloids 503 of huntingtin-exon1 show different cytotoxicity. Proceedings of the National 504 Academy of Sciences 106, 9679-9684 (2009). 
50518 Sun, C.-S. et al. Conformational switch of polyglutamine-expanded huntingtin into benign aggregates leads to neuroprotective effect. Sci Rep 5, 14992, doi:10.1038/srep14992 (2015).

50819 Nucifora, F. C. et al. Interference by huntingtin and atrophin-1 with cbp-mediated 509 transcription leading to cellular toxicity. Science 291, 2423-2428, 510 doi:10.1126/science.1056784 (2001).

51120 Albin, R. L. Polyglutamine inclusion body toxicity. Mov Disord 32, 1686, 512 doi:10.1002/mds.27226 (2017).

51321 Scherzinger, E. et al. Self-assembly of polyglutamine-containing huntingtin 514 fragments into amyloid-like fibrils: implications for Huntingtons disease pathology.

515 Proceedings of the National Academy of Sciences 96, 4604-4609 (1999).

51622 Bugg, C. W., Isas, J. M., Fischer, T., Patterson, P. H. \& Langen, R. Structural 517 features and domain organization of huntingtin fibrils. J Biol Chem 287, 31739$518 \quad 31746$, doi:10.1074/jbc.M112.353839 (2012).

51923 Crick, S. L., Ruff, K. M., Garai, K., Frieden, C. \& Pappu, R. V. Unmasking the roles 520 of $\mathrm{N}$ - and $\mathrm{C}$-terminal flanking sequences from exon 1 of huntingtin as modulators 521 of polyglutamine aggregation. Proc Natl Acad Sci U S A 110, 20075-20080, 522 doi:10.1073/pnas.1320626110 (2013).

52324 Gruber, A. et al. Molecular and structural architecture of polyQ aggregates in yeast. 524 Proc Natl Acad Sci U S A 115, E3446-E3453, doi:10.1073/pnas.1717978115 525 (2018).

52625 Bäuerlein, F. J. B. et al. In Situ Architecture and Cellular Interactions of PolyQ $527 \quad$ Inclusions. Cell 171, 179-187.e110, doi:10.1016/j.cell.2017.08.009 (2017). 
52826 Warner, J. B. et al. Monomeric Huntingtin Exon 1 Has Similar Overall Structural Features for Wild-Type and Pathological Polyglutamine Lengths. J Am Chem Soc 139, 14456-14469, doi:10.1021/jacs.7b06659 (2017).

53127 Galaz-Montoya, J. G. \& Ludtke, S. J. The advent of structural biology in situby single particle cryo-electron tomography. Biophys Rep 3, 17-35, doi:10.1007/s41048-017-0040-0 (2017).

53428 Zhang, P. Advances in cryo-electron tomography and subtomogram averaging and classification. Curr Opin Struct Biol 58, 249-258, doi:10.1016/j.sbi.2019.05.021 (2019).

53729 Shahmoradian, S. H. et al. TRiCs tricks inhibit huntingtin aggregation. eLife 2, $538 \quad$ e00710 (2013).

53930 Darrow, M. C. et al. Structural Mechanisms of Mutant Huntingtin Aggregation 540 Suppression by the Synthetic Chaperonin-like CCT5 Complex Explained by 541 Cryoelectron Tomography. Journal of Biological Chemistry 290, 17451-17461 $542 \quad$ (2015).

54331 Shen, K. et al. Control of the structural landscape and neuronal proteotoxicity of 544 mutant Huntingtin by domains flanking the polyQ tract. Elife 5, 545 doi:10.7554/eLife.18065 (2016).

54632 Deng, Y. et al. ICON: 3D reconstruction with 'missing-information' restoration in 547 biological electron tomography. Journal of Structural Biology 195, 100-112, $548 \quad$ doi:10.1016/j.jsb.2016.04.004 (2016).

54933 Chen, Y. et al. Accelerating electron tomography reconstruction algorithm ICON 550 with GPU. Biophys Rep 3, 36-42, doi:10.1007/s41048-017-0041-z (2017). 
55134 Chen, M. et al. Convolutional neural networks for automated annotation of cellular cryo-electron tomograms. Nat Methods 14, 983-985, doi:10.1038/nmeth.4405 (2017).

55435 Chen, M. et al. A complete data processing workflow for cryo-ET and

37 Galaz-Montoya, J. G., Flanagan, J., Schmid, M. F. \& Ludtke, S. J. Single particle

56138 Dobson, C. M. The structural basis of protein folding and its links with human disease. Philos Trans $R$ Soc Lond B Biol Sci 356, 133-145,

56439 Maiuri, T., Woloshansky, T., Xia, J. \& Truant, R. The huntingtin N17 domain is a 565

56740 Scherzinger, E. et al. Huntingtin-encoded polyglutamine expansions form amyloid568 like protein aggregates in vitro and in vivo. Cell 90, 549-558, doi:10.1016/s0092-

57041 Chen, S., Berthelier, V., Yang, W. \& Wetzel, R. Polyglutamine aggregation 571 behavior in vitro supports a recruitment mechanism of cytotoxicity. J Mol Biol 311, 173-182, doi:10.1006/jmbi.2001.4850 (2001). 
57342 Chen, S., Berthelier, V., Hamilton, J. B., O'Nuallain, B. \& Wetzel, R. Amyloid-like features of polyglutamine aggregates and their assembly kinetics. Biochemistry 41, 7391-7399, doi:10.1021/bi011772q (2002).

57643 Fitzpatrick, A. W. P. et al. Cryo-EM structures of tau filaments from Alzheimer's disease. Nature 547, 185-190, doi:10.1038/nature23002 (2017).

57844 Gremer, L. et al. Fibril structure of amyloid-beta(1-42) by cryo-electron microscopy. Science 358, 116-119, doi:10.1126/science.aao2825 (2017).

58045 Guerrero-Ferreira, R. et al. Cryo-EM structure of alpha-synuclein fibrils. Elife 7, 581 doi:10.7554/eLife.36402 (2018).

$58246 \mathrm{Li}$, B. et al. Cryo-EM of full-length alpha-synuclein reveals fibril polymorphs with a 583 common structural kernel. Nat Commun 9, 3609, doi:10.1038/s41467-018-059712 (2018).

$58547 \mathrm{Li}$, Y. et al. Amyloid fibril structure of alpha-synuclein determined by cryo-electron 586 microscopy. Cell Res 28, 897-903, doi:10.1038/s41422-018-0075-x (2018).

58748 ladanza, M. G., Jackson, M. P., Hewitt, E. W., Ranson, N. A. \& Radford, S. E. A 588 new era for understanding amyloid structures and disease. Nat Rev Mol Cell Biol 19, 755-773, doi:10.1038/s41580-018-0060-8 (2018).

59049 Fitzpatrick, A. W. \& Saibil, H. R. Cryo-EM of amyloid fibrils and cellular aggregates. $591 \quad$ Curr Opin Struct Biol 58, 34-42, doi:10.1016/j.sbi.2019.05.003 (2019).

59250 Scheres, S. H., Zhang, W., Falcon, B. \& Goedert, M. Cryo-EM structures of tau 593 filaments. Curr Opin Struct Biol 64, 17-25, doi:10.1016/j.sbi.2020.05.011 (2020).

59451 Wetzel, R. Physical chemistry of polyglutamine: intriguing tales of a monotonous 595 sequence. J Mol Biol 421, 466-490, doi:10.1016/j.jmb.2012.01.030 (2012). 
59652 Ruggeri, F. S. et al. Nanoscale studies link amyloid maturity with polyglutamine 597 diseases onset. Sci Rep 6, 31155, doi:10.1038/srep31155 (2016).

59853 Jensen, E. C. Use of fluorescent probes: their effect on cell biology and limitations. Anat Rec (Hoboken) 295, 2031-2036, doi:10.1002/ar.22602 (2012).

60054 Rigort, A. et al. Automated segmentation of electron tomograms for a quantitative 601 description of actin filament networks. J Struct Biol 177, 135-144, 602

60355 Rusu, M., Starosolski, Z., Wahle, M., Rigort, A. \& Wriggers, W. Automated tracing 604 of filaments in 3D electron tomography reconstructions using Sculptor and Situs. 605 J Struct Biol 178, 121-128, doi:10.1016/j.jsb.2012.03.001 (2012).

60656 Yu, Z. \& Frangakis, A. S. M-free: scoring the reference bias in sub-tomogram 607 averaging and template matching. $J$ Struct Biol 187, 10-19, $608 \quad$ doi:10.1016/j.jsb.2014.05.007 (2014).

60957 Hecksel, C. W. et al. Quantifying Variability of Manual Annotation in Cryo-Electron 610 Tomograms. Microscopy and microanalysis: the official journal of Microscopy 611 Society of America, Microbeam Analysis Society, Microscopical Society of $612 \quad$ Canada, 1-10 (2016).

61358 Wagner, A. S. et al. Self-assembly of Mutant Huntingtin Exon-1 Fragments into 614 Large Complex Fibrillar Structures Involves Nucleated Branching. J Mol Biol 430, $615 \quad$ 1725-1744, doi:10.1016/j.jmb.2018.03.017 (2018).

61659 Lu, K., Jacob, J., Thiyagarajan, P., Conticello, V. P. \& Lynn, D. G. Exploiting 617 amyloid fibril lamination for nanotube self-assembly. J Am Chem Soc 125, 6391618 6393, doi:10.1021/ja0341642 (2003). 
61960 Poirier, M. A. et al. Huntingtin spheroids and protofibrils as precursors in polyglutamine fibrilization. J Biol Chem 277, 41032-41037, doi:10.1074/jbc.M205809200 (2002).

62261 Schneider, R. et al. Structural characterization of polyglutamine fibrils by solidstate NMR spectroscopy. J Mol Biol 412, 121-136, doi:10.1016/j.jmb.2011.06.045 (2011).

62562 Duim, W. C., Chen, B., Frydman, J. \& Moerner, W. E. Sub-diffraction imaging of huntingtin protein aggregates by fluorescence blink-microscopy and atomic force microscopy. Chemphyschem 12, 2387-2390, doi:10.1002/cphc.201100392 (2011).

62963 Duim, W. C., Jiang, Y., Shen, K., Frydman, J. \& Moerner, W. E. Super-resolution fluorescence of huntingtin reveals growth of globular species into short fibers and coexistence of distinct aggregates. ACS Chem Biol 9, 2767-2778, doi:10.1021/cb500335w (2014).

63665 Hoop, C. L. et al. Polyglutamine amyloid core boundaries and flanking domain 637 dynamics in huntingtin fragment fibrils determined by solid-state nuclear magnetic 638 resonance. Biochemistry 53, 6653-6666, doi:10.1021/bi501010q (2014).

63966 Hoop, C. L. et al. Huntingtin exon 1 fibrils feature an interdigitated $\beta$-hairpin-based 640 polyglutamine core. Proc Natl Acad Sci U S A 113, 1546-1551, 641 doi:10.1073/pnas.1521933113 (2016). 
64267 Lin, H.-K. et al. Fibril polymorphism affects immobilized non-amyloid flanking domains of huntingtin exon1 rather than its polyglutamine core. Nat Commun $\mathbf{8}$, 15462, doi:10.1038/ncomms15462 (2017).

64568 Vieweg, S., Ansaloni, A., Wang, Z. M., Warner, J. B. \& Lashuel, H. A. An Intein646 based Strategy for the Production of Tag-free Huntingtin Exon 1 Proteins Enables 647 New Insights into the Polyglutamine Dependence of Httex1 Aggregation and Fibril 648 Formation. J Biol Chem 291, 12074-12086, doi:10.1074/jbc.M116.713982 (2016).

64969 Adegbuyiro, A., Sedighi, F., Pilkington, A. W. t., Groover, S. \& Legleiter, J. Proteins 650 Containing Expanded Polyglutamine Tracts and Neurodegenerative Disease. 651 Biochemistry 56, 1199-1217, doi:10.1021/acs.biochem.6b00936 (2017).

65270 Hegde, R. N. et al. TBK1 phosphorylates mutant Huntingtin and suppresses its 653 aggregation and toxicity in Huntington's disease models. EMBO J 39, e104671, 654 doi:10.15252/embj.2020104671 (2020).

65571 Mastronarde, D. N. Automated electron microscope tomography using robust 656 prediction of specimen movements. Journal of structural biology 152, 36-51 657 (2005).

65872 Kremer, J. R., Mastronarde, D. N. \& Mclntosh, J. R. Computer visualization of 659 three-dimensional image data using IMOD. Journal of structural biology 116, 71$66076(1996)$.

66173 Radermacher, M. Three-Dimensional reconstruction of single particles from 662 random and nonrandom tilt series. Journal of Electron Microscopy Technique $\mathbf{9}$, 663 359-394 (1988). 
66474 Iwasaki, K. et al. Electron tomography reveals diverse conformations of integrin $665 \quad$ allbß3 in the active state. Journal of structural biology 150, 259-267 (2005).

66675 Bartesaghi, A. et al. Classification and 3D averaging with missing wedge correction 667 in biological electron tomography. Journal of structural biology 162, 436-450 $668 \quad$ (2008).

66976 Zhang, L. \& Ren, G. IPET and FETR: experimental approach for studying 670 molecular structure dynamics by cryo-electron tomography of a single-molecule 671 structure. PloS one 7, e30249, doi:10.1371/journal.pone.0030249 (2012).

67277 Galaz-Montoya, J. G. et al. Alignment algorithms and per-particle CTF correction 673 for single particle cryo-electron tomography. Journal of structural biology 194, 383$674 \quad 394(2016)$.

67578 Pettersen, E. F. et al. UCSF Chimera--a visualization system for exploratory 676 research and analysis. J Comput Chem 25, 1605-1612, doi:10.1002/jcc.20084 677 (2004).

678 


\section{Figures}

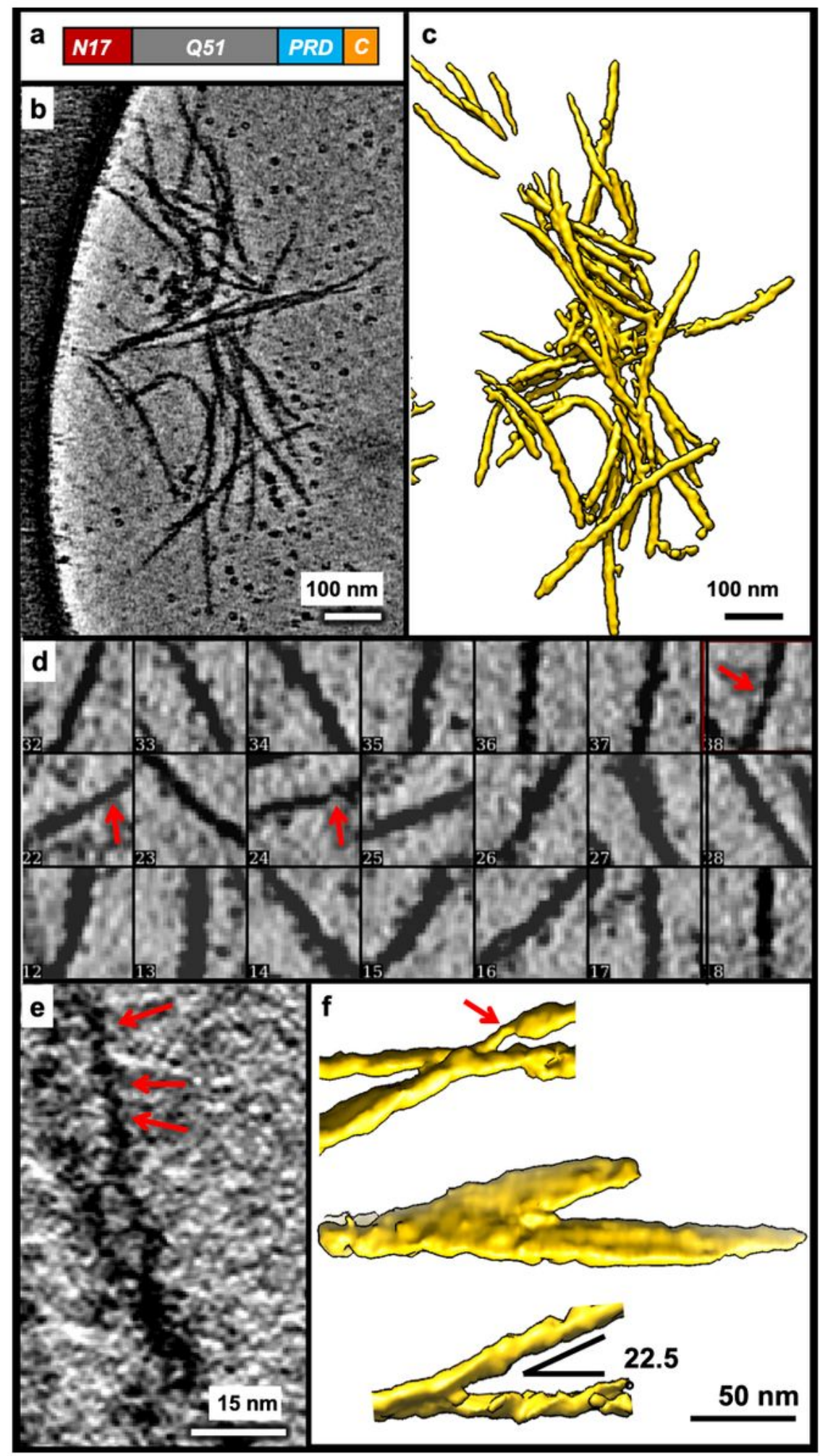

Figure 1

MEx1-Q51 filaments exhibit a large variation in width within and across filaments. (a) Schematic of the mEx1-Q51 construct. (b) Slice parallel to $x y(\sim 1.7 \mathrm{~nm}$ thick) through a representative $4 x$ down-sampled cryoET tomogram of aggregated $\mathrm{mEx} 1-\mathrm{Q} 51$, reconstructed with compressed sensing, lightly filtered to 
enhance visualization, and (c) corresponding semi-automated 3D annotation. (d) Selected areas from slices of large mEx1-Q51 aggregates showing individual filaments, widely varying in width, with the thinnest filaments exhibiting regions down to $\sim 2 \mathrm{~nm}$ width, indicated by the redarrows. (e) Zoomed-in view of a xy slice ( $\sim .4 \mathrm{~nm}$ thick) from a selected region of a tomogram without any down-sampling, showcasing ultra-thin regions in mEx1-Q51 filaments. (f) Sections of annotated mEX1- Q51 filamentous aggregates from cryoET tomograms showing relatively narrow branching angles and an example of a thicker laminated sheet-like region (the annotation example in the middle).

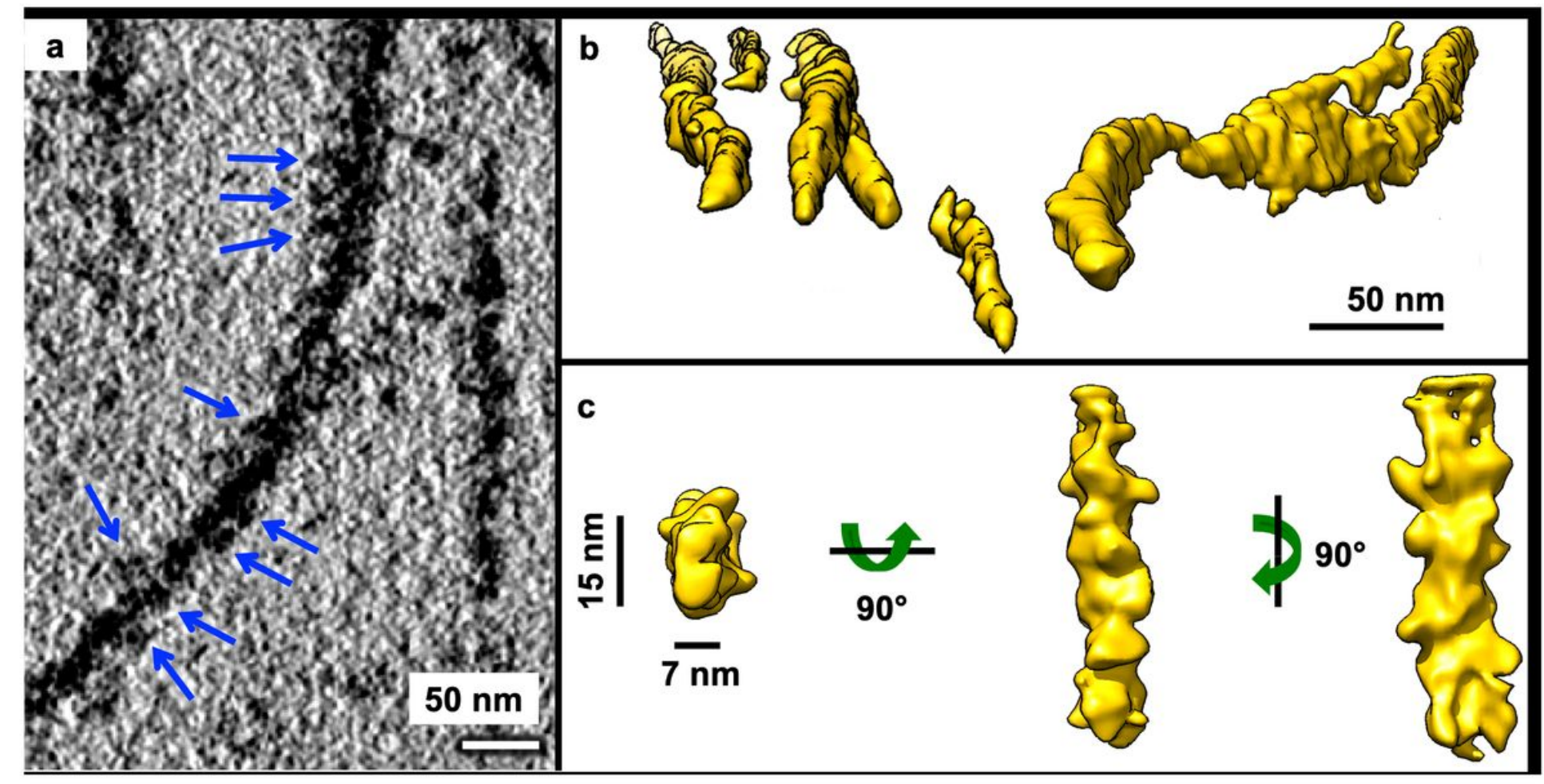

Figure 2

Aggregated mEx1-Q51 exhibits lumpy, slab-shaped filaments. (a) Pseudo periodic pattern of repeating lumps (blue arrows) along the length of an mEx1-Q51 filament as seen in an xy slice (4.4 A thick) from a tomogram of aggregated mEx1-Q51. (b) Selected regions from semi-automated neural network annotations showing lumpy filaments of various widths, including sheet-like regions (middle region of right-most example). (c) Subtomogram average of a subpopulation of filament segments exhibiting a lumpy $7 \times 15 \mathrm{~nm}$ slab-shaped morphology. 


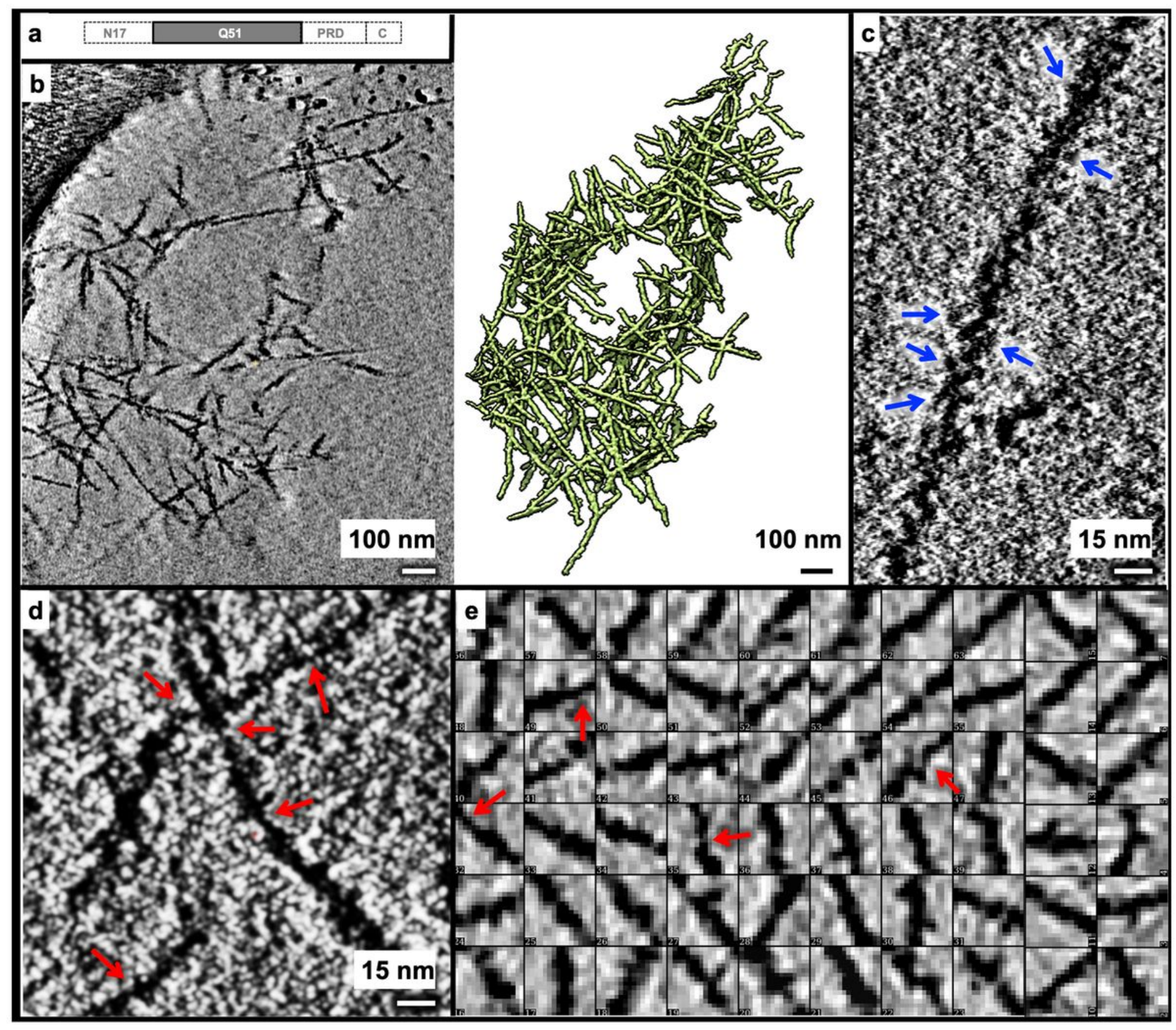

Figure 3

Lumpy Q51 filaments exhibit a large range of widths. (a) Schematic of the Q51 construct, lacking all mEx1 domains except for the polyQ tract. (b) Slice parallel to the xy plane $(\sim 2.1 \mathrm{~nm}$ thick) through a representative 4x down-sampled cryoET tomogram of aggregated Q51 reconstructed with compressed sensing (left) and corresponding 3D annotation (right). Zoomed-in views of xy slices ( 0.5 nm thick) from selected regions of the tomogram shown in A but without any down-sampling, exhibiting (c) a pseudo periodic pattern of repeating lumps along the length of a Q51 filament (blue arrows), and (d) regions in thin filaments that are as thin as $\sim 2 \mathrm{~nm}$ in width (red arrows). (e) Examples of 2D xy slices through representative 3D subtomograms of Q51 filaments showing a wide variation in width, including super-thin regions $\sim 2 \mathrm{~nm}$ in width (red arrows). 

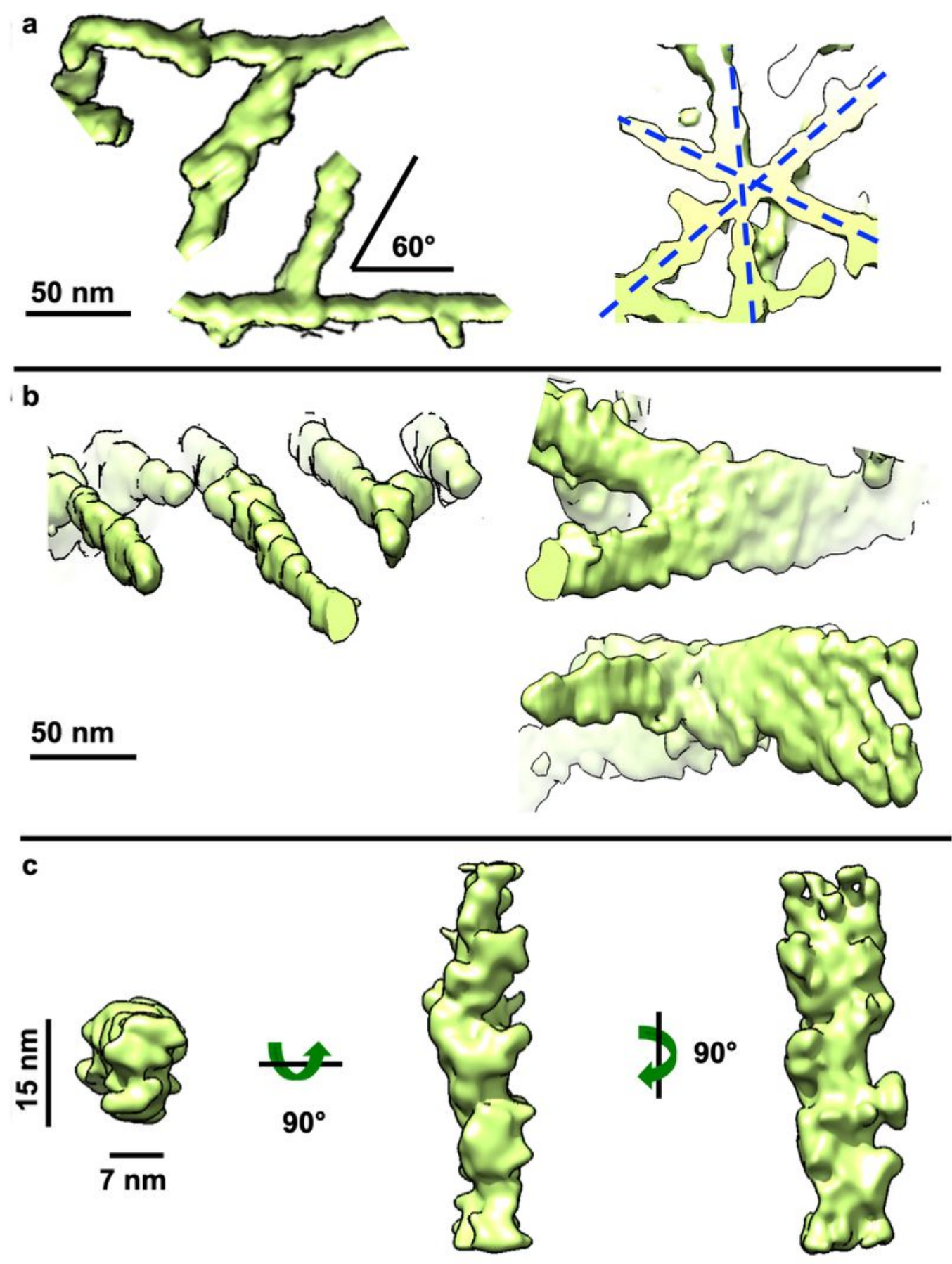

Figure 4

Aggregated Q51 exhibits lamination sheets and predominantly lumpy,slab-shaped filaments. (a) Representative sections of annotated Q51 filamentous aggregates from cryoET tomograms showing their most common branching/crossover angle $\left(\sim 60^{\circ}\right)$, often in an asterisk like pattern, and (b) thicker regions (right) akin to lamination, onside thinner ones (left). (c) Subtomogram average of a subpopulation of filament segments exhibiting a lumpy $7 \times 15 \mathrm{~nm}$ slab-shaped morphology. 


\section{Supplementary Files}

This is a list of supplementary files associated with this preprint. Click to download.

- rs.pdf 